\title{
Ergodicity for the Navier-Stokes Equation with Degenerate Random Forcing: Finite-Dimensional Approximation
}

\author{
WEINAN E \\ Princeton University \\ AND \\ JONATHAN C. MATTINGLY \\ Stanford University
}

\begin{abstract}
We study Galerkin truncations of the two-dimensional Navier-Stokes equation under degenerate, large-scale, stochastic forcing. We identify the minimal set of modes that has to be forced in order for the system to be ergodic. Our results rely heavily on the structure of the nonlinearity. (C) 2001 John Wiley \& Sons, Inc.
\end{abstract}

\section{Introduction and Main Result}

One basic assumption in the theory of turbulence is that of ergodicity: Under large-scale forcing, energy is transferred through nonlinearity to the small scales and the system establishes a unique statistical steady state. In numerical simulations, one typically forces very few low modes when studying the direct cascade process. Statistical properties of the turbulent flows are measured or calculated through time averaging rather than ensemble averaging. The main purpose of our work is to rigorously establish the validity of this basic assumption. We find it convenient to study this problem in a stochastic setting. Consider the two-dimensional Navier-Stokes equation with a stochastic forcing

$$
\left\{\begin{array}{l}
\frac{\partial \mathbf{u}}{\partial t}+(\mathbf{u} \cdot \nabla) \mathbf{u}+\nabla p=v \Delta \mathbf{u}+\frac{d \mathbf{F}}{d t} \\
\nabla \cdot \mathbf{u}=0 .
\end{array}\right.
$$

For simplicity we will work with the periodic boundary conditions on the domain $[0,2 \pi]^{2}$. This two-dimensional torus will be denoted by $\mathbb{T}^{2}$. It is more convenient to work with the vorticity

$$
\omega=\frac{\partial u_{2}}{\partial x_{1}}-\frac{\partial u_{1}}{\partial x_{2}}=\nabla^{\perp} \cdot \mathbf{u}, \quad \nabla^{\perp}=\left(-\frac{\partial}{\partial x_{2}}, \frac{\partial}{\partial x_{1}}\right) .
$$


Then (1.1) can be expressed as

$$
\frac{\partial \omega}{\partial t}+(\mathbf{u} \cdot \nabla) \omega=v \Delta \omega+\frac{d f}{d t}
$$

with $d f=\nabla^{\perp} \cdot d \mathbf{F}$. We will consider the case when the stochastic forcing has the following form:

$$
d f=\sum_{\mathbf{k} \in K} \sigma_{\mathbf{k}} \cos (\mathbf{k} \cdot \mathbf{x}) d B_{\mathbf{k}}(t)+\sum_{\mathbf{k} \in K} \gamma_{\mathbf{k}} \sin (\mathbf{k} \cdot \mathbf{x}) d W_{\mathbf{k}}(t)
$$

where the $B_{\mathbf{k}}$ 's and $W_{\mathbf{k}}$ 's are independent standard Wiener processes defined on a probability space $(\Omega, \mathcal{F}, \mathbb{P})$. Expectations on this probability space will be denoted by $\mathbb{E}$. $\sigma_{\mathbf{k}}$ and $\gamma_{\mathbf{k}}$ are positive constants representing the amplitude of the forcing. $K$ is the set of modes that are forced. We will primarily be interested in the case when $K$ contains very few low modes. We limit ourselves to the situation when there is no mean flow. Hence we assume for the remainder that $(0,0) \notin K$.

Our main purpose is to identify the minimal condition in order for the system to have a unique invariant measure. Writing $\omega(\mathbf{x}, t)=\sum_{\mathbf{k}} \alpha_{\mathbf{k}}(t) \cos (\mathbf{k} \cdot \mathbf{x})+$ $\sum_{\mathbf{k}} \beta_{\mathbf{k}}(t) \sin (\mathbf{k} \cdot \mathbf{x})$ where the index $\mathbf{k}$ is in the first quadrant, we can rewrite (1.3) as

$$
\begin{aligned}
d \alpha_{\mathbf{l}}= & \left\{-v|\mathbf{I}|^{2} \alpha_{\mathbf{l}}+\sum_{\mathbf{j}+\mathbf{k}=\mathbf{l}} \frac{\mathbf{j}^{\perp} \cdot \mathbf{k}}{|\mathbf{j}|^{2}}\left(-\alpha_{\mathbf{j}} \alpha_{\mathbf{k}}+\beta_{\mathbf{j}} \beta_{\mathbf{k}}\right)\right. \\
& \left.+\sum_{\mathbf{j}-\mathbf{k}=\mathbf{l}} \frac{\mathbf{j}^{\perp} \cdot \mathbf{k}}{|\mathbf{j}|^{2}}\left(\alpha_{\mathbf{j}} \alpha_{\mathbf{k}}+\beta_{\mathbf{j}} \beta_{\mathbf{k}}\right)\right\} d t+\sigma_{\mathbf{l}} d B_{\mathbf{l}} \\
d \beta_{\mathbf{l}}= & \left\{-v|\mathbf{l}|^{2} \beta_{\mathbf{l}}-\sum_{\mathbf{j}+\mathbf{k}=\mathbf{l}} \frac{\mathbf{j}^{\perp} \cdot \mathbf{k}}{|\mathbf{j}|^{2}}\left(\alpha_{\mathbf{j}} \beta_{\mathbf{k}}+\beta_{\mathbf{j}} \alpha_{\mathbf{k}}\right)\right. \\
& \left.+\sum_{\mathbf{j}-\mathbf{k}=\mathbf{l}} \frac{\mathbf{j}^{\perp} \cdot \mathbf{k}}{|\mathbf{j}|^{2}}\left(-\alpha_{\mathbf{j}} \beta_{\mathbf{k}}+\beta_{\mathbf{j}} \alpha_{\mathbf{k}}\right)\right\} d t+\gamma_{\mathbf{l}} d W_{\mathbf{l}}
\end{aligned}
$$

where $\mathbf{j}^{\perp}=\left(-j_{2}, j_{1}\right)$. Here and in the following, we adopt the convention that the summation is done only over terms in which the indices $\mathbf{j}, \mathbf{k}$, and $\mathbf{l}$ are in the first quadrant. We will use $\|\cdot\|$ to denote the $L^{2}$-norm on $\mathbb{T}^{2}$. Of course $\|\omega\|^{2}=$ $\sum \alpha_{\mathbf{k}}^{2}+\beta_{\mathbf{k}}^{2}$ is the enstrophy. In this paper, we will consider finite-dimensional approximations of (1.4) and (1.5):

$$
\begin{aligned}
d \alpha_{\mathbf{l}}= & \left\{-v|\mathbf{l}|^{2} \alpha_{\mathbf{l}}+\sum_{\mathbf{j}+\mathbf{k}=\mathbf{l}}^{N} \frac{\mathbf{j}^{\perp} \cdot \mathbf{k}}{|\mathbf{j}|^{2}}\left(-\alpha_{\mathbf{j}} \alpha_{\mathbf{k}}+\beta_{\mathbf{j}} \beta_{\mathbf{k}}\right)\right. \\
& \left.+\sum_{\mathbf{j}-\mathbf{k}=\mathbf{l}}^{N} \frac{\mathbf{j}^{\perp} \cdot \mathbf{k}}{|\mathbf{j}|^{2}}\left(\alpha_{\mathbf{j}} \alpha_{\mathbf{k}}+\beta_{\mathbf{j}} \beta_{\mathbf{k}}\right)\right\} d t+\sigma_{\mathbf{l}} d B_{\mathbf{l}}
\end{aligned}
$$




$$
\begin{aligned}
d \beta_{\mathbf{I}}= & \left\{-v|\mathbf{I}|^{2} \beta_{\mathbf{l}}-\sum_{\mathbf{j}+\mathbf{k}=\mathbf{l}}^{N} \frac{\mathbf{j}^{\perp} \cdot \mathbf{k}}{|\mathbf{j}|^{2}}\left(\alpha_{\mathbf{j}} \beta_{\mathbf{k}}+\beta_{\mathbf{j}} \alpha_{\mathbf{k}}\right)\right. \\
& \left.+\sum_{\mathbf{j}-\mathbf{k}=\mathbf{l}}^{N} \frac{\mathbf{j}^{\perp} \cdot \mathbf{k}}{|\mathbf{j}|^{2}}\left(-\alpha_{\mathbf{j}} \beta_{\mathbf{k}}+\beta_{\mathbf{j}} \alpha_{\mathbf{k}}\right)\right\} d t+\gamma_{\mathbf{l}} d W_{\mathbf{l}}
\end{aligned}
$$

where $\sum^{N}$ means that the sum is over first quadrant indices $\mathbf{j}, \mathbf{k}$, and $\mathbf{l}$ such that $|\mathbf{j}|_{\infty},|\mathbf{k}|_{\infty},|\mathbf{l}|_{\infty} \leq N$.

Just as (1.4) and (1.5) give the evolution of the Fourier modes $\alpha_{\mathbf{k}}$ and $\beta_{\mathbf{k}}$ of a function $\omega(x, t)$ that solves (1.2), we can associate a function $\omega^{(N)}(x, t)$ whose Fourier modes solve $\left(1.4_{N}\right)$ and $\left(1.5_{N}\right)$. One sees that $\omega^{(N)}$ satisfies the equation

$$
\frac{\partial \omega^{(N)}}{\partial t}+P_{N}\left(\mathbf{u}^{(N)} \cdot \nabla\right) \omega^{(N)}=v \Delta \omega^{(N)}+\frac{d f^{(N)}}{d t} .
$$

Here $\mathcal{P}_{N}$ is the projection operator onto the Fourier modes less than or equal to $N$ and $f^{(N)}=\mathcal{P}_{N} f$ and $\mathbf{u}^{(N)}=\mathcal{P}_{N} \mathbf{u}$. From now on we will restrict our attention to $\left(2_{N}\right),\left(4_{N}\right)$, and $\left(5_{N}\right)$ and omit the superscript $N .\left(1.4_{N}\right)$ and $\left(1.5_{N}\right)$ define a diffusion process on $\mathbb{R}^{(N+1)^{2}-1}$. In counting the dimension of the state space, we exclude the $(0,0)$ mode, as there is no mean flow. By an invariant measure $\pi$, we mean a probability measure that solves

$$
\pi(A)=\int P_{t}(\omega, A) \pi(d \omega)
$$

for all measurable sets $A$ and $t>0$. Here $P_{t}\left(\omega_{0}, A\right)=\mathbb{P}_{\omega_{0}}\{\omega(t) \in A\}$ is the transition kernel for the Markov process $\left(1.4_{N}\right)-\left(1.5_{N}\right)$.

Our main result is the following:

THEOREM 1.1 Let $K_{1}=\{(0,1),(1,1)\}$ and $K_{2}=\{(1,0),(1,1)\}$. If $K \supset K_{1}$ or $K \supset K_{2}$, then $\left(1.4_{N}\right)$ and $\left(1.5_{N}\right)$ have a unique invariant measure $\pi$.

The existence of an invariant measure follows from standard compactness arguments. The tightness needed to yield compactness is implied by a priori bounds on the enstrophy. Because the existence of an invariant measure is covered by results in the appendix, we do not dwell on it here. The proof of uniqueness uses the following result of Harris [10]: Let $\left\{x_{n}, n=0,1, \ldots\right\}$ be a Markov process on a topological space $\mathbb{X}$ with Borel $\sigma$-algebra $\mathfrak{B}$. The Markov process $\left\{x_{n}, n=0,1, \ldots\right\}$ is said to satisfy Harris' condition if there exists a $\sigma$-finite measure $m$ on $\mathbb{X}$ such that if $m(E)>0, E \in \mathfrak{B}$, then

$$
\mathbb{P}_{x_{0}}\left\{x_{n} \in E \text { infinitely often }\right\}=1
$$

for all starting points $x_{0}$ in $\mathbb{X}$. Under this condition Harris proved that there is a measure $Q$, unique up to a constant multiplier, that solves the equation

$$
Q(E)=\int_{\mathbb{X}} P(x, E) Q(d x) \quad \text { for } E \in \mathfrak{B}
$$


where $P(x, \cdot)$ is the transition probability distribution of the Markov process.

Our task is reduced to showing that $\left(1.4_{N}\right)$ and $\left(1.5_{N}\right)$ satisfy Harris' condition. This is done by proving the following:

(1) Starting from any initial position, the dynamics enters any neighborhood of the origin infinitely often.

(2) The transition probability distribution has a smooth density.

The main idea for proving (1) is to observe that in the absence of forcing, solutions of $\left(1.4_{N}\right)$ and $\left(1.5_{N}\right)$ decay exponentially fast due to the viscous term. This decay still holds when the forcing is small. The main idea for proving (2) is to prove that the Fokker-Planck operator associated with $\left(1.4_{N}\right)-\left(1.5_{N}\right)$ is hypoelliptic. Since the work of Harris, there has been extensive work extending his basic result to continuous-time Markov processes [9, 13, 16, 22]. In particular, it is proven in [1, 9] that a diffusion process satisfying the continuous analogue of Harris' condition has at most one invariant probability measure.

The following two lemmas, proved later, will be used to establish that our diffusion process satisfies Harris' condition:

Lemma 1.2 Assume that $K_{1} \subset K$ or $K_{2} \subset K$. Then the Markov process $\left(1.4_{N}\right)-$ $\left(1.5_{N}\right)$ has a transition density $p_{t}(x, y)$ that is $C^{\infty}$ in $(x, y, t)$ for $t>0$.

LEMMA 1.3 Fix an $h>0$ and an open neighborhood $U_{0}$ of the origin. Then given any initial condition $\omega_{0} \in \mathbb{R}^{(N+1)^{2}-1}$,

$$
\mathbb{P}_{\omega_{0}}\left\{\omega(n h) \in U_{0} \text { for infinitely many } n\right\}=1 .
$$

By using these lemmas, ergodicity follows quickly.

Proof of Theorem 1.1: To prove uniqueness, it is enough to show that the chain obtained by sampling the Markov process at fixed time intervals has a unique invariant measure.

Fix a $h>0$ and let 0 denote the origin. Since $\int p_{h}(0, y) d y=1$, there exists a $y_{0} \in \mathbb{R}^{(N+1)^{2}-1}$ such that $p_{h}\left(0, y_{0}\right)>0$. By the regularity of $p_{h}(x, y)$, there exists an open neighborhood of 0 , denoted by $A_{1}$, an open neighborhood of $y_{0}$, denoted by $A_{2}$, and a positive constant $\delta_{0}$ such that

$$
p_{h}(x, y)>\delta_{0} \quad \text { if } x \in A_{1}, y \in A_{2} .
$$

Let $m$ be the normalized Lebesgue measure on $A_{2}$. We claim that the Markov chain $\omega_{n}$ obtained from $\left(1.4_{N}\right)-\left(1.5_{N}\right)$ by setting $\omega_{n}=\omega(n h)$ satisfies Harris' condition. In other words, we need to show that for any measurable subset $B$ of $A_{2}$ such that $m(B)>0$,

$$
\mathbb{P}_{\omega_{0}}\left\{\omega_{n} \in B \text { for infinitely many } n\right\}=1 .
$$

To see this, let $t_{n}$ be the $n^{\text {th }}$ time that $\left\{\omega_{n}\right\}$ is in $A_{1}$. By Lemma 1.3, we see that the $t_{n}$ are well-defined and finite with probability 1 . Define $\#_{B}(n)$ to be the number of 
$k \in[0, n]$ such that $\omega_{k} \in B$. First observe that since $B \subset A_{2}$,

$$
\mathbb{P}_{\omega_{0}}\left\{\omega_{n} \in B \mid \omega_{n-1} \in A_{1}\right\}=\int_{B} p_{h}\left(\omega_{n-1}, y\right) d y \geq \delta_{0} m(B) .
$$

Since $m(B)>0$, we know that $\delta_{1}=\delta_{0} m(B)>0$. Now fix some positive $M$ and $n$ with $n>M$. In light of the above calculation, $\mathbb{P}\left\{\#_{B}\left(t_{n+1}\right)<M\right\} \leq\left(1-\delta_{1}\right)^{n-M}$. Since $t_{n} \rightarrow \infty$ as $n \rightarrow \infty$, we see that $\mathbb{P}\left\{\#_{B}(n)<M\right\} \rightarrow 0$ as $n \rightarrow \infty$. Since $M$ was arbitrary, $B$ is visited infinitely many times almost surely. This implies that the invariant measure must be unique. This completes the proof of Theorem 1.1.

Theorem 1.1 together with the ergodic theorem implies that the time averages of the empirical measures converge to the unique invariant measure. This convergence can be improved. In fact, we can prove the following:

THEOREM 1.4 Under the same assumption as Theorem 1.1, the unique invariant measure $\pi$ guaranteed by Theorem 1.1 is exponentially attracting. More precisely, there exist fixed positive constants $B$ and $r$ so that

$$
\sup _{A \in \mathfrak{A}}\left|P_{t}\left(\omega_{0}, A\right)-\pi(A)\right| \leq B\left[1+\left\|\omega_{0}\right\|^{2}\right] e^{-r t}
$$

where $\omega_{0}=\sum \alpha_{\mathbf{k}}(0) \cos (\mathbf{k} \cdot \mathbf{x})+\beta_{\mathbf{k}}(0) \sin (\mathbf{k} \cdot \mathbf{x})$ and $\mathfrak{A}$ is the measurable subsets of $\mathbb{R}^{(N+1)^{2}-1}$.

It is important to remark that the constants $B$ and $r$ depend on the order of the approximation $N$ used to define $\left(1.4_{N}\right)$ and $\left(1.5_{N}\right)$. The proof of this result, and an even stronger one, is given in the appendix. Even though our proof of Theorem 1.4 will imply Theorem 1.1, we give a separate proof of Theorem 1.1. We do this in hopes of highlighting the central issues in proving ergodicity in such a system without the complications of extracting a convergence rate.

Before we end this section, let us remark that the same results can also be extended with little change to the system

$$
\omega_{t}+(\mathbf{u} \cdot \nabla) \omega=-\alpha \omega+f
$$

where $\alpha$ is a fixed positive constant. In fact, when considering finite-dimensional Galerkin truncations of these systems, the friction term $-\alpha \omega$ has the same effect as the viscous term $v \Delta \omega$. The importance of considering frictional damping in studying two-dimensional turbulence is discussed in [5].

\section{Regularity of the Transition Density}

$\left(1.4_{N}\right)-\left(1.5_{N}\right)$ is a degenerate diffusion process with analytic coefficients. The regularity of the transition density of such processes can be studied using Malliavin 
calculus or more classical methods. If we write the Fokker-Planck operator (the generator) of a diffusion process in the form

$$
L=X_{0}+\frac{1}{2} \sum_{j=1}^{l} X_{j}^{2}
$$

then the transition density is smooth if at each point of the state space $\mathbb{R}^{(N+1)^{2}-1}$

$$
\begin{array}{ll}
X_{j}, & j=1,2, \ldots, l, \\
{\left[X_{j}, X_{k}\right],} & j, k=0,1, \ldots, l, \\
{\left[X_{j},\left[X_{k_{1}}, X_{k_{2}}\right]\right],} & j, k_{1}, k_{2}=0,1, \ldots, l,
\end{array}
$$

span the tangent space $[12,15,17,23]$. Notice that we allow the first-order terms to enter into the higher-order brackets. This condition can be restated as follows: The ideal generated by $X_{1}, X_{2}, \ldots, X_{l}$ in the Lie algebra generated $X_{0}, X_{1}, X_{2}, \ldots, X_{l}$ must have full rank at every point. We denote by $\operatorname{span}(\omega)$ the ideal generated by $X_{1}, X_{2}, \ldots, X_{l}$ at the point $\omega$ in $\mathbb{R}^{(N+1)^{2}-1}$. The Fokker-Planck operator for $\left(1.4_{N}\right)-\left(1.5_{N}\right)$ can be written as

$$
L=X_{0}+\frac{1}{2} \sum_{\mathbf{k} \in K}^{N} \sigma_{\mathbf{k}}^{2}\left(\frac{\partial}{\partial \alpha_{\mathbf{k}}}\right)^{2}+\frac{1}{2} \sum_{\mathbf{k} \in K}^{N} \gamma_{\mathbf{k}}^{2}\left(\frac{\partial}{\partial \beta_{\mathbf{k}}}\right)^{2}
$$

where

$$
\begin{aligned}
X_{0}=\sum_{\mathbf{l}}^{N}\left\{\sum_{\mathbf{j}+\mathbf{k}=\mathbf{l}}^{N} \frac{\mathbf{j}^{\perp} \cdot \mathbf{k}}{|\mathbf{j}|^{2}}\left(-\alpha_{\mathbf{j}} \alpha_{\mathbf{k}}+\beta_{\mathbf{j}} \alpha_{\mathbf{k}}\right)\right. \\
\left.+\sum_{\mathbf{j}=\mathbf{k}=\mathbf{l}}^{N} \frac{\mathbf{j}^{\perp} \cdot \mathbf{k}}{|\mathbf{j}|^{2}}\left(\alpha_{\mathbf{j}} \alpha_{\mathbf{k}}+\beta_{\mathbf{j}} \beta_{\mathbf{k}}\right)-v|\mathbf{l}|^{2} \alpha_{\mathbf{l}}\right\} \frac{\partial}{\partial \alpha_{\mathbf{l}}} \\
+\sum_{\mathbf{l}}^{N}\left\{\sum_{\mathbf{j}+\mathbf{k}=\mathbf{l}}^{N} \frac{\mathbf{j}^{\perp} \cdot \mathbf{k}}{|\mathbf{j}|^{2}}\left(-\alpha_{\mathbf{j}} \beta_{\mathbf{k}}-\beta_{\mathbf{j}} \alpha_{\mathbf{k}}\right)\right. \\
\left.\quad+\sum_{\mathbf{j}-\mathbf{k}=\mathbf{l}}^{N} \frac{\mathbf{j}^{\perp} \cdot \mathbf{k}}{|\mathbf{j}|^{2}}\left(-\alpha_{\mathbf{j}} \beta_{\mathbf{k}}+\beta_{\mathbf{j}} \alpha_{\mathbf{k}}\right)-v|\mathbf{l}|^{2} \beta_{\mathbf{l}}\right\} \frac{\partial}{\partial \beta_{\mathbf{l}}} .
\end{aligned}
$$


We can now calculate

$$
\begin{aligned}
& X_{\mathbf{m}}=\left[X_{0}, \frac{\partial}{\partial \alpha_{\mathbf{m}}}\right] \\
& =\sum_{\mathbf{l}}^{N}\left\{\alpha_{\mathbf{l}-\mathbf{m}}\left(\frac{1}{|\mathbf{m}|^{2}}-\frac{1}{|\mathbf{I}-\mathbf{m}|^{2}}\right)+\alpha_{\mathbf{m}-\mathbf{I}} \frac{1}{|\mathbf{m}|^{2}}+\alpha_{\mathbf{l}+\mathbf{m}} \frac{1}{|\mathbf{I}+\mathbf{m}|^{2}}\right\}\left(\mathbf{l}^{\perp} \cdot \mathbf{m}\right) \frac{\partial}{\partial \alpha_{\mathbf{l}}} \\
& +\sum_{\mathbf{l}}^{N}\left\{\beta_{\mathbf{l}-\mathbf{m}}\left(\frac{1}{|\mathbf{m}|^{2}}-\frac{1}{|\mathbf{I}-\mathbf{m}|^{2}}\right)-\beta_{\mathbf{m}-\mathbf{l}} \frac{1}{|\mathbf{m}|^{2}}+\beta_{\mathbf{l}+\mathbf{m}} \frac{1}{|\mathbf{I}+\mathbf{m}|^{2}}\right\}\left(\mathbf{I}^{\perp} \cdot \mathbf{m}\right) \frac{\partial}{\partial \beta_{\mathbf{l}}} \\
& -v|\mathbf{m}|^{2} \frac{\partial}{\partial \alpha_{\mathbf{m}}} \\
& Y_{\mathbf{m}}=\left[X_{0}, \frac{\partial}{\partial \beta_{\mathbf{m}}}\right] \\
& =\sum_{\mathbf{l}}^{N}\left\{\beta_{\mathbf{I}-\mathbf{m}}\left(-\frac{1}{|\mathbf{m}|^{2}}+\frac{1}{|\mathbf{I}-\mathbf{m}|^{2}}\right)+\beta_{\mathbf{m}-\mathbf{l}} \frac{1}{|\mathbf{m}|^{2}}+\beta_{\mathbf{m}+\mathbf{l}} \frac{1}{|\mathbf{I}+\mathbf{m}|^{2}}\right\}\left(\mathbf{l}^{\perp} \cdot \mathbf{m}\right) \frac{\partial}{\partial \alpha_{\mathbf{l}}} \\
& +\sum_{\mathbf{I}}^{N}\left\{\alpha_{\mathbf{l}-\mathbf{m}}\left(\frac{1}{|\mathbf{m}|^{2}}-\frac{1}{|\mathbf{I}-\mathbf{m}|^{2}}\right)+\alpha_{\mathbf{m}-\mathbf{I}} \frac{1}{|\mathbf{m}|^{2}}-\alpha_{\mathbf{m}+\mathbf{l}} \frac{1}{|\mathbf{I}+\mathbf{m}|^{2}}\right\}\left(\mathbf{I}^{\perp} \cdot \mathbf{m}\right) \frac{\partial}{\partial \beta_{\mathbf{l}}} \\
& -v|\mathbf{m}|^{2} \frac{\partial}{\partial \beta_{\mathbf{m}}}
\end{aligned}
$$

We emphasize our convention that the terms are present in the sums only if the indices are in the first quadrant. If we denote by

$$
Q_{N}=\left\{\mathbf{j}=\left(j_{1}, j_{2}\right), 0 \leq j_{1}, j_{2} \leq N,\left(j_{1}, j_{2}\right) \neq(0,0)\right\},
$$

then the first sum in $X_{\mathbf{m}}$ should be written as

$$
\begin{aligned}
& \sum_{\mathbf{l}, \mathbf{I}-\mathbf{m} \in Q_{N}} \alpha_{\mathbf{l}-\mathbf{m}}\left(\frac{1}{|\mathbf{m}|^{2}}-\frac{1}{|\mathbf{I}-\mathbf{m}|^{2}}\right)\left(\mathbf{l}^{\perp} \cdot \mathbf{m}\right) \frac{\partial}{\partial \alpha_{\mathbf{l}}} \\
& \quad+\sum_{\mathbf{l}, \mathbf{m}-\mathbf{l} \in Q_{N}} \alpha_{\mathbf{m}-\mathbf{l}} \frac{1}{|\mathbf{m}|^{2}}\left(\mathbf{I}^{\perp} \cdot \mathbf{m}\right) \frac{\partial}{\partial \alpha_{\mathbf{l}}}+\sum_{\mathbf{l}, \mathbf{I}+\mathbf{m} \in Q_{N}} \alpha_{\mathbf{l}+\mathbf{m}} \frac{1}{|\mathbf{I}+\mathbf{m}|^{2}}\left(\mathbf{I}^{\perp} \cdot \mathbf{m}\right) \frac{\partial}{\partial \alpha_{\mathbf{l}}}
\end{aligned}
$$

and similarly for the other terms. We then have

$$
\begin{aligned}
& {\left[X_{\mathbf{m}}, \frac{\partial}{\partial \beta_{\mathbf{k}}}\right]=\left(\mathbf{k}^{\perp} \cdot \mathbf{m}\right)\left\{\left(\frac{1}{|\mathbf{m}|^{2}}-\frac{1}{|\mathbf{k}|^{2}}\right) \frac{\partial}{\partial \beta_{\mathbf{m}+\mathbf{k}}}+\frac{1}{|\mathbf{m}|^{2}} \frac{\partial}{\partial \beta_{\mathbf{m}-\mathbf{k}}}+\frac{1}{|\mathbf{k}|^{2}} \frac{\partial}{\partial \beta_{\mathbf{k}-\mathbf{m}}}\right\},} \\
& {\left[X_{\mathbf{m}}, \frac{\partial}{\partial \alpha_{\mathbf{k}}}\right]=\left(\mathbf{k}^{\perp} \cdot \mathbf{m}\right)\left\{\left(\frac{1}{|\mathbf{m}|^{2}}-\frac{1}{|\mathbf{k}|^{2}}\right) \frac{\partial}{\partial \alpha_{\mathbf{m}+\mathbf{k}}}-\frac{1}{|\mathbf{m}|^{2}} \frac{\partial}{\partial \alpha_{\mathbf{m}-\mathbf{k}}}+\frac{1}{|\mathbf{k}|^{2}} \frac{\partial}{\partial \alpha_{\mathbf{k}-\mathbf{m}}}\right\},}
\end{aligned}
$$




$$
\begin{aligned}
& {\left[Y_{\mathbf{m}}, \frac{\partial}{\partial \beta_{\mathbf{k}}}\right]=\left(\mathbf{k}^{\perp} \cdot \mathbf{m}\right)\left\{-\left(\frac{1}{|\mathbf{m}|^{2}}-\frac{1}{|\mathbf{k}|^{2}}\right) \frac{\partial}{\partial \alpha_{\mathbf{m}+\mathbf{k}}}-\frac{1}{|\mathbf{m}|^{2}} \frac{\partial}{\partial \alpha_{\mathbf{m}-\mathbf{k}}}+\frac{1}{|\mathbf{k}|^{2}} \frac{\partial}{\partial \alpha_{\mathbf{k}-\mathbf{m}}}\right\},} \\
& {\left[Y_{\mathbf{m}}, \frac{\partial}{\partial \alpha_{\mathbf{k}}}\right]=\left(\mathbf{k}^{\perp} \cdot \mathbf{m}\right)\left\{\left(\frac{1}{|\mathbf{m}|^{2}}-\frac{1}{|\mathbf{k}|^{2}}\right) \frac{\partial}{\partial \beta_{\mathbf{m}+\mathbf{k}}}-\frac{1}{|\mathbf{m}|^{2}} \frac{\partial}{\partial \beta_{\mathbf{m}-\mathbf{k}}}-\frac{1}{|\mathbf{k}|^{2}} \frac{\partial}{\partial \beta_{\mathbf{k}-\mathbf{m}}}\right\} .}
\end{aligned}
$$

Again the same summation convention as we discussed above applies. Fix any $\omega \in \mathbb{R}^{(N+1)^{2}-1}$. Take $\mathbf{m}=(1,1)$ and $\mathbf{k}=(0,1)$; then we have

$$
\left[X_{\mathbf{m}}, \frac{\partial}{\partial \alpha_{\mathbf{k}}}\right]=\frac{1}{2}\left\{\frac{\partial}{\partial \alpha_{(1,2)}}+\frac{\partial}{\partial \alpha_{(1,0)}}\right\}, \quad\left[Y_{\mathbf{m}}, \frac{\partial}{\partial \beta_{\mathbf{k}}}\right]=-\frac{1}{2}\left\{\frac{\partial}{\partial \alpha_{(1,2)}}-\frac{\partial}{\partial \alpha_{(1,0)}}\right\} .
$$

Therefore if $K_{1} \subset K$, then

$$
\frac{\partial}{\partial \alpha_{(1,2)}}, \frac{\partial}{\partial \alpha_{(1,0)}} \in \operatorname{span}(\omega)
$$

Similarly, take $\mathbf{k}=(1,0)$ and $\mathbf{m}=(1,2)$; we find $\partial / \partial \alpha_{(0,2)} \in \operatorname{span}(\omega)$. Proceeding in this way, we find

$$
\frac{\partial}{\partial \alpha_{(1, l)}}, \frac{\partial}{\partial \alpha_{(0, l)}} \in \operatorname{span}(\omega) \quad \text { for } l \leq N .
$$

Take $\mathbf{k}=(1,0)$ and $\mathbf{m}=(1, l)$; we get $\frac{\partial}{\partial \alpha_{(2, l)}} \in \operatorname{span}(\omega)$ for $l>0$. Take $\mathbf{k}=(2,1)$ and $\mathbf{m}=(0,1)$; we get $\partial / \partial \alpha_{(2,0)} \in \operatorname{span}(\omega)$. By induction, we obtain

$$
\frac{\partial}{\partial \alpha_{(k, l)}}, \frac{\partial}{\partial \beta_{(k, l)}} \in \operatorname{span}(\omega) \quad \text { for all } 0 \leq k, l \leq N,(k, l) \neq(0,0) .
$$

This proves Lemma 1.1 in the case when $K_{1} \subset K$. The case when $K_{2} \subset K$ is proved in the same way.

Another application of Malliavain calculus to the study of invariant measures of stochastic PDEs can be found in [6].

\section{Recurrence of Neighborhoods of the Origin}

The main result of this section is the proof of Lemma 1.3. It will be based on two auxiliary lemmas given below. The first says that if one waits long enough, the chance of entering any neighborhood of the origin from any initial condition in a compact ball is uniformly bounded away from zero. The second says that this compact ball can be chosen so that the process enters it regularly. These ideas are made precise by Lemmas 3.1 and 3.2, respectively.

We begin by clarifying our setting and notation. Recall that $\left(1.4_{N}\right)$ and $\left(1.5_{N}\right)$ are defined relative to some level of approximation $N$. As this level of approximation will play no explicit role in this section, we will write $\omega(t)$ for $\omega^{(N)}(t)$ to alleviate notational clutter. In fact, if $\mathbb{E}\|\Delta f\|<\infty$, then the proofs in this section hold uniformly in $N$ and even hold for the true solution to (1.2). Defining

$$
\mathscr{B}(c)=\left\{g \in L^{2}\left(\mathbb{T}^{2}\right):\|g\|=\left(\int_{\mathbb{T}^{2}}|g(\mathbf{x})|^{2} d \mathbf{x}\right)^{\frac{1}{2}} \leq c\right\},
$$


we prove the following:

LEMMA 3.1 Let $\mathscr{B}_{0}=\mathscr{B}\left(C_{0}\right)$ and $\mathcal{B}_{1}=\mathscr{B}\left(C_{1}\right)$ be two arbitrary balls about the origin and $h$ be some positive constant. Then there exists a $T_{0}=T_{0}\left(C_{0}, C_{1}\right)>0$ so that for any $T \geq T_{0}$ there is a $p^{*}$ with

$$
\inf _{\omega_{0} \in \mathcal{B}_{0}} \mathbb{P}_{\omega_{0}}\left\{\omega(t) \in \mathscr{B}_{1} \text { for all } t \in[T, T+h]\right\} \geq p^{*}>0 .
$$

We emphasize that $p^{*}$ is a positive constant depending only on $T, h, C_{0}$, and $C_{1}$.

The above lemma reflects the fact that the origin is the global attractor of the unforced dynamics. The next lemma reflects the fact that enstrophy is a classical Lyapunov function for the system when forced with a fixed deterministic body forcing. In the stochastic setting, this has the interpretation that outside of some ball the dynamics moves inward on average. We will make this observation more precise in the appendix. For the moment we need only the following result:

LemMA 3.2 If $\left\|\omega_{0}\right\|>C$ a.s. for some $C$, with $C^{2}>\varepsilon_{1} / 2 v, \varepsilon_{1}=\sum_{\mathbf{k}}\left(\sigma_{\mathbf{k}}^{2}+\gamma_{\mathbf{k}}^{2}\right)$, then

$$
\mathbb{P}\left\{\tau_{\mathcal{C}}\left(\omega_{0}\right) \geq t\right\} \leq \frac{\mathbb{E}\left\{\left\|\omega_{0}\right\|^{2}\right\}}{C^{2}} \exp (-2 \nu \delta t)
$$

where $\delta=1-\varepsilon_{1} / 2 v C^{2}, \mathcal{C}=\mathscr{B}(C)$, and

$$
\tau_{\mathcal{C}}\left(\omega_{0}\right)=\inf \left\{s>0: \omega(s) \in \mathcal{C} \text { given } \omega(0)=\omega_{0}\right\} .
$$

Before proving the above two lemmas, we use them to prove Lemma 1.3.

Proof of Lemma 1.3 : Define $C$ and $C$ as in Lemma 3.2. Since $U_{1}$ is open, we can pick a $C_{1}$ small enough so that $\mathscr{B}_{1}=\mathscr{B}\left(C_{1}\right) \subset U_{1}$. Now let $T_{0}$ be the constant given by Lemma 3.1 when $\mathscr{B}_{0}=\mathcal{C}$ and $h$ is taken to be the sampling rate given in the statement of Lemma 1.3. Now let $T$ be the smallest integer multiple of $h$ that is greater than $\left(T_{0}+2 h\right)$ and set $n^{*}=T / h$. (Notice that by construction $n^{*} \geq 2$.) Define $\omega_{n}$ by $\omega(n h)$. By Lemma 3.1 there exists a $p^{*}>0$ so that

$$
\mathbb{P}\left\{\omega_{n+n^{*}-1} \in U_{1} \mid \omega(t) \in \mathcal{C} \text { for some } t \in[(n-1) h, n h]\right\} \geq p^{*} .
$$

Define the sequence of increasing integer stopping times $\tau_{n}$ by

$$
\tau_{0}=\inf \{n \geq 1: \omega(t) \in \mathcal{C} \text { for some } t \in[(n-1) h, n h]\}
$$

and for $k>0$

$$
\tau_{k}=\inf \left\{n \geq \tau_{k-1}+\left(n^{*}+1\right): \omega(t) \in \mathcal{C} \text { for some } t \in[(n-1) h, n h]\right\} .
$$

By Lemma 3.2, it is clear that each $\tau_{k}$ is almost surely finite. As in the proof of Lemma 1.3, define $\#_{U_{1}}(n)$ as the number of $k \in[0, n]$ so that $\omega_{n} \in U_{1}$. By (3.1), we have that for any $n$ and $M$, with $M<n, \mathbb{P}\left\{\#_{U_{1}}\left(\tau_{n}+n^{*}\right)>M\right\} \leq\left(1-p^{*}\right)^{n-M}$. By the same reasoning used in the proof of Lemma 1.3, we see that $U_{1}$ is visited infinitely often almost surely. 
Proof of Lemma 3.1: Define $v(t)=\omega(t)-\hat{f}(t)$ where $\hat{f}(t)=f(t)-f(0)$. Here $f(t)$ is the Brownian motion defined in (1.3), which takes values in analytic functions on the two-dimensioinal torus. Using (1.2), we see that $v(t)$ satisfies

$$
\frac{\partial v}{\partial t}=v \Delta v-\mathcal{P}_{N}(\mathbf{u} \cdot \nabla)(v+\hat{f}) .
$$

Taking the $L^{2}$-inner product of this equation with $v$ and recalling that

$$
\int v(\mathbf{x})(u(\mathbf{x}) \cdot \nabla) v(\mathbf{x}) d \mathbf{x}=0
$$

produces

$$
\frac{1}{2} \frac{d}{d t}\|v\|^{2}=-v\|\nabla v\|^{2}-\int_{\mathbb{T}^{2}} v(\mathbf{x})(\mathbf{u}(\mathbf{x}) \cdot \nabla) \hat{f}(\mathbf{x}) d \mathbf{x} .
$$

By standard estimates on the nonlinear term (see [3]) and the fact we are on the torus, we have

$$
\left|\int v(\mathbf{x})(u(\mathbf{x}) \cdot \nabla) \hat{f}(\mathbf{x}) d \mathbf{x}\right| \leq C_{3}\|v\|\|\nabla u\|\|\Delta \hat{f}\| .
$$

Since $\|\nabla u\|=\|\omega\|$ and $\omega=v+\hat{f}$, the above estimate gives

$$
\begin{aligned}
\frac{1}{2} \frac{d}{d t}\|v\|^{2} & \leq-v\|\nabla v\|^{2}+C_{3}\|v\|\|v+f\|\|\Delta \hat{f}\| \\
& \leq-v\|\nabla v\|^{2}+C_{3}\|v\|^{2}\|\Delta \hat{f}\|+C_{3}\|v\|\|\hat{f}\|\|\Delta \hat{f}\| .
\end{aligned}
$$

Using the Poincaré inequality, $\|v\|^{2} \leq\|\nabla v\|^{2}$, we get

$$
\frac{1}{2} \frac{d}{d t}\|v\|^{2} \leq-\left(\frac{v}{2}-C_{3}\|\Delta \hat{f}\|\right)\|v\|^{2}+\frac{C_{3}^{2}}{2 v}\|\hat{f}\|^{2}\|\Delta \hat{f}\|^{2} .
$$

Fix any $\delta>0$ and define for any $T>0$

$$
\Omega^{\prime}(\delta, T)=\left\{g \in C\left([0, T+h] ; L^{2}\left(\mathbb{T}^{2}\right)\right): \sup _{s \in[0, T]}\|\Delta g(s)\| \leq \min \left(\delta, \frac{v}{4 C_{3}}\right)\right\} .
$$

If $f \in \Omega^{\prime}$, then there exists a constant $C_{4}$ depending only on the domain so that

$$
\|v(t)\|^{2} \leq\|v(0)\|^{2} e^{-\frac{v}{2} t}+\frac{C_{4}}{(v)^{2}} \min \left(\delta, \frac{v}{4 C_{3}}\right)^{4} .
$$

Hence if $\|\omega(0)\|<C_{0}$, then given any $C_{1}>0$ there exists a $T$ and a $\delta$ such that $\|v(T)\|<C_{1} / 2$. By possibly decreasing $\delta$, we can assume that $\|f(t)\|<C_{1} / 2$ for $t \in[T, T+h]$ if $f \in \Omega^{\prime}$. Putting everything together, we have that for appropriate $T$ and $\delta$,

$$
\|\omega(0)\| \leq C_{0} \quad \text { and } \quad f \in \Omega^{\prime}(\delta, T) \Longrightarrow\|\omega(t)\| \leq\|v(t)\|+\|f(t)\| \leq C_{1}
$$

for $t \in[T, T+h]$. Since for any $T \in(0, \infty)$ and $\delta_{0}>0, \Omega^{\prime}$ is an open set in the supremum topology, we know that $\mathbb{P}\left\{\Omega^{\prime}\right\}>0$. 
Proof of Lemma A.1: Define

$$
Y(s, \omega)=e^{2 \delta \nu s}\|\omega(s)\|^{2}=e^{2 \delta \nu s}\left(\sum_{\mathbf{k}}\left|\alpha_{\mathbf{k}}\right|^{2}+\sum_{\mathbf{k}}\left|\beta_{\mathbf{k}}\right|^{2}\right) .
$$

Applying Itô's formula to the map $\omega \mapsto Y(s, \omega)$ gives

$d Y(s)=\left[2 \delta v Y(s)-2 v e^{\delta \nu s}\|\nabla \omega(s)\|^{2}+e^{2 \delta \nu s} \mathcal{E}_{1}\right] d s+2 e^{2 \delta \nu s} \int_{\mathbb{T}^{2}} \omega(x, s) \cdot d f(x, s) d x$

where $\varepsilon_{1}=\sum_{\mathbf{k}}\left(\left|\sigma_{\mathbf{k}}\right|^{2}+\left|\gamma_{\mathbf{k}}\right|^{2}\right)$ and

$$
\int_{\mathbb{T}^{2}} \omega(x, s) \cdot d f(x, s) d x=\sum_{\mathbf{k}} \alpha_{\mathbf{k}} d B_{\mathbf{k}}(s)+\sum_{\mathbf{k}} \beta_{\mathbf{k}} d W_{\mathbf{k}}(s) .
$$

Using the Poincaré inequality on the second term allows us to obtain

$$
d Y(s) \leq\left[\mathcal{E}_{1}-2 v(1-\delta)\|\omega(s)\|^{2}\right] e^{2 \delta v s} d s+2 e^{2 \delta \nu s} \int_{\mathbb{T}^{2}} \omega(x, s) \cdot d f(x, s) d x .
$$

Integrating up to some stopping time $T$ and observing that by definition $2 v(1-\delta)=$ $\varepsilon_{1} / C^{2}$ produces

$$
\begin{aligned}
\mathbb{E} Y(T) \leq & \mathbb{E} Y(0)+\mathcal{E}_{1} \mathbb{E} e^{2 \delta v T} \int_{0}^{T}\left[1-\frac{\|\omega(s)\|^{2}}{C^{2}}\right] d s \\
& +\mathbb{E} \int_{0}^{T} e^{2 \delta v s} \int_{\mathbb{T}^{2}} \omega(x, s) \cdot d f(x, s) d x .
\end{aligned}
$$

For any $n>1$, define $S_{n}=\inf \left\{s>0:\|\omega(s)\|^{2} \geq n\|\omega(0)\|^{2}\right\}$. Fix a $t>0$ and set $T=\tau_{\mathcal{C}} \wedge S_{n} \wedge t$. (Here $a \wedge b=\min (a, b)$.) Observe that the optional stopping time lemma implies that

$$
\begin{aligned}
\mathbb{E} \int_{0}^{T} e^{2 \delta v s} \int_{\mathbb{T}^{2}} \omega(x, s) \cdot d f(x, s) d x & =\mathbb{E} \int_{0}^{T} e^{2 \delta \nu s} \int_{\mathbb{T}^{2}} \omega\left(x, s \wedge S_{n}\right) \cdot d f(x, s) d x \\
& =0
\end{aligned}
$$

because the martingale is now a bounded martingale and the stopping time a bounded stopping time. Furthermore, for $s \leq \tau_{C}$ we know that $\|\omega(s)\|>C$, and hence the second term on the right-hand side of (3.3) can be neglected. This produces $\mathbb{E} Y(T) \leq \mathbb{E} Y(0)$. Since $\|\omega(s)\|^{2}$ is almost surely finite and continuous in time, $S_{n} \rightarrow \infty$ as $n \rightarrow \infty$. Hence taking $n \rightarrow \infty$ gives $\mathbb{E} Y\left(t \wedge \tau_{\mathcal{C}}\right) \leq \mathbb{E} Y(0)$. Next observe that

$$
\begin{aligned}
\mathbb{E} Y(0) & \geq \mathbb{E}\left\{Y\left(\tau_{\mathcal{C}}\right) \mid t>\tau_{\mathcal{C}}\right\} \mathbb{P}\left\{t>\tau_{\mathcal{C}}\right\}+\mathbb{E}\left\{Y(t) \mid t \leq \tau_{\mathcal{C}}\right\} \mathbb{P}\left\{t \leq \tau_{\mathcal{C}}\right\} \\
& \geq \mathbb{E}\left\{Y(t) \mid t \leq \tau_{\mathcal{C}}\right\} \mathbb{P}\left\{t \leq \tau_{\mathcal{C}}\right\} \\
& \geq C^{2} e^{2 v \delta t} \mathbb{P}\left\{t \leq \tau_{\mathcal{C}}\right\}
\end{aligned}
$$


Rearranging things gives the result.

\section{Concluding Remarks}

Several interesting questions remain. The most obvious is the question whether the result continues to hold under the same assumption for the full Navier-Stokes equation. While we strongly believe this is so, we have not yet succeeded in giving a proof. In fact, for the full Navier-Stokes equation, the only existing result so far for the uniqueness of an invariant measure under degenerate noise is for the case when all determining modes are forced $[2,4,14]$. The number of determining modes goes to infinity as $v \rightarrow 0$. Previous results on uniqueness of invariant measures $[7,8]$ require that all modes be forced. The second question is whether the condition in Theorem 1.1 is sharp. We suspect in the case of Kolmogorov flow when only the mode $(1,0)$ or $(0,1)$ is forced, the invariant measure should not be unique at high enough Reynolds number. For small Reynolds numbers, there is a unique invariant measure regardless of the structure of the forcing [18]. It is not clear what happens if both modes $(1,0)$ and $(0,1)$ are forced. Next one might ask whether the bracket calculation correctly captures the rate of energy transfer. Work on this problem is in progress.

\section{Appendix: Exponential Convergence}

We say that $V: \mathbb{R}^{d} \rightarrow[0, \infty)$ is a Lyapunov function for a diffusion $X_{t}$ with generator $L$ if the following hold:

(1) $V(\mathbf{x}) \rightarrow \infty$ as $|\mathbf{x}| \rightarrow \infty$.

(2) The level sets $\{\mathbf{x}: V(\mathbf{x}) \leq c\}$ are precompact for all positive $c$.

(3) There exist positive constants $a$ and $b$ and a compact set $C$ so that

$$
L V(\mathbf{x})<-a V(\mathbf{x})+b \mathbf{1}_{C}(\mathbf{x}) \text { for all } \mathbf{x} .
$$

The existence of a Lyapunov function will serve us in two ways. First, it is more than enough to prove that the process is bounded in probability. This in turn provides the tightness that gives the existence of invariant measures. Second, it ensures the positive recurrence needed to ensure uniqueness. In fact, it ensures a recurrence regular enough to give exponential mixing.

LEMMA A.1 The enstrophy is a Lyapunov function for system $\left(1.4_{N}\right)$ and $\left(1.5_{N}\right)$. More precisely, consider $L$ defined by (2.2) and $\left\{\left(\alpha_{\mathbf{k}}, \beta_{\mathbf{k}}\right)\right\}_{|\mathbf{k}| \leq N}$ solving $\left(1.4_{N}\right)$ and $\left(1.5_{N}\right)$. If $V\left(\alpha_{\mathbf{k}}, \beta_{\mathbf{k}}\right)=\sum_{\mathbf{k}}\left(\alpha_{\mathbf{k}}^{2}+\beta_{\mathbf{k}}^{2}\right)$, then

$$
L V \leq-2 v V+\varepsilon_{1}
$$

where $\varepsilon_{1}=\sum_{\mathbf{k}}\left(\sigma_{\mathbf{k}}^{2}+\gamma_{\mathbf{k}}^{2}\right)$. 
Observe that this implies that enstrophy is a Lyapunov function with the set $C$ defined by

$$
C=\left\{\left(\alpha_{\mathbf{k}}, \beta_{\mathbf{k}}\right)_{|\mathbf{k}| \leq N}:\left[\sum_{\mathbf{k}}\left(\alpha_{\mathbf{k}}^{2}+\beta_{\mathbf{k}}^{2}\right)\right]^{\frac{1}{2}} \leq \frac{\mathcal{E}_{1}}{2 v-\epsilon}\right\},
$$

$a=2 v-\epsilon$, and $b=\varepsilon_{1}$ where $\epsilon$ is any number in $(0,2 v)$. Of course, $C$ can be viewed as a set in $\mathcal{P}_{N} L^{2}\left(\mathbb{T}^{2}\right)$ given by

$$
C=\left\{g \in \mathcal{P}_{N} L^{2}\left(\mathbb{T}^{2}\right):\|g\| \leq \frac{\mathcal{E}_{1}}{2 v-\epsilon}\right\} .
$$

Proof of Lemma A.1: This is just the standard enstrophy estimate. By direct calculation and using the fact that the nonlinearity disappears because of (3.2), we obtain

$$
\begin{aligned}
L \sum_{\mathbf{k}}\left(\alpha_{\mathbf{k}}^{2}+\beta_{\mathbf{k}}^{2}\right) & =-2 v \sum_{\mathbf{k}}|\mathbf{k}|^{2}\left(\alpha_{\mathbf{k}}^{2}+\beta_{\mathbf{k}}^{2}\right)+\sum_{\mathbf{k}}\left(\sigma_{\mathbf{k}}^{2}+\gamma_{\mathbf{k}}^{2}\right) \\
& \leq-2 v \sum_{\mathbf{k}}\left(\alpha_{\mathbf{k}}^{2}+\beta_{\mathbf{k}}^{2}\right)+\sum_{\mathbf{k}}\left(\sigma_{\mathbf{k}}^{2}+\gamma_{\mathbf{k}}^{2}\right)
\end{aligned}
$$

THEOREM A.2 Let $X_{t}$ be a diffusion on $\mathbb{R}^{d}$ satisfying the hypoellipticity condition (H). Suppose the following additional conditions hold:

(i) There exists a function $V: \mathbb{R}^{d} \rightarrow \mathbb{R}^{+}$that is a Lyapunov function, in the sense of (L), for the diffusion $X_{t}$.

(ii) There exists a point $x^{*}$ such that for any open neighborhood $U$ of $x^{*}$, the following property holds: There exists a sampling rate $h>0$ so that for any $x \in \mathbb{R}^{d}$ there exists an $n>0$ with

$$
\mathbb{P}_{x}\left\{X_{n h} \in U\right\}>0 .
$$

Then the diffusion $X_{t}$ has an invariant measure $\pi$, and this measure is unique. Furthermore, there exists positive $B$ and $r$ so that for all $x$

$$
\left\|P_{t}(x, \cdot)-\pi\right\|_{T V} \leq\left\|P_{t}(x, \cdot)-\pi\right\|_{V} \leq B[1+V(x)] e^{-r t} .
$$

Here $\|\cdot\|_{T V}$ is the standard total variation norm and $\|\cdot\|_{V}$ is the weight total-variational norm given by

$$
\left\|P_{t}(x, \cdot)-\pi\right\|_{V}=\sup _{f \in \mathcal{V}}\left|\int f(y) P_{t}(x, d y)-\int f(y) \pi(d y)\right|
$$

with $\mathcal{V}=\{$ measurable $f$ with $|f(x)|<1+V(x)\}$.

PROOF OF THEOREMS 1.1 AND 1.4: We only need to show that the assumptions of Theorem A.2 are satisfied. Hypoellipticity was shown in Section 2. The needed Lyapunov function is given by Lemma A.1. Taking the origin as the distinguished point $x^{*}$, the last condition is implied by Lemma 3.1. 
Now we turn to the proof of Theorem A.2. With a little additional work, this theorem follows from [10, 13], or [20, 21, 22]. Since [20, 21, 22] are closest to our setup, we will connect with the theorems contained there. These works apply to a much more general setting and the reader should not think that the proof of Theorem A.2 is overly involved. Once the the assumptions of Theorem A.2 are established, a direct proof of the theorem is rather straightforward. If one proceeds along the lines of Lemma 1.3 but with more care, one can control the frequency of visits to neighborhoods of the origin. From this one can estimate the rate of convergence; see [19], for example.

Since realizing such an analysis does require some setup, we opt instead to connect with existing theorems in the literature. To make contact with the results in $[20,21,22]$, we need a few definitions and preliminary results. Consider the Markov chain obtained from $X_{t}$ by sampling at time intervals $h$. We set $X_{n}^{h}=$ $X_{n h}$. Recall the definition of $\varphi$-irreducibility. $X_{n}^{h}$ is $\varphi$-irreducible if there exists a nontrivial, finite measure $\varphi$ such that the following holds for any initial $x$ and measurable set $B$ :

$$
\varphi(B)>0 \Longrightarrow \mathbb{P}_{x}\left\{X_{n}^{h} \in B\right\}>0 \text { for some } n>0 .
$$

The following lemma will be the bridge between the measure-theoretic irreducibility defined above and the more topological irreducibility implied by the results of Section 3.

Lemma A.3 Let $X_{t}$ be a diffusion in $\mathbb{R}^{d}$ satisfying $(\mathrm{H})$. Then for any $t>0$ and $x^{\prime} \in \mathbb{R}^{d}$, there exist a ball $\mathcal{B}_{1}$ about $x^{\prime}$, a measurable set $A$, and constant $\delta \in(0,1)$ such that

$$
\inf _{x \in \mathscr{B}_{1}} P_{t}(x, \cdot) \geq \delta m_{A}(\cdot) .
$$

Here $m_{A}(\cdot)$ is the Lebesgue measure restricted to the set $A$ and normalized to 1. In other words, $m_{A}(A)=1$ and $m_{A}\left(A^{c}\right)=0$.

Proof of Lemma A.3: By hypoellipticity, we know that

$$
P_{t}(x, B)=\int_{B} p_{t}(x, y) m(d y)
$$

where $m$ is Lebesgue measure an $p_{t}(x, y)$ is $C^{\infty}$ as a function in $(x, y)$. Since $P_{t}\left(x^{\prime}, \mathbb{R}^{d}\right)=1$, there must be some $y_{0}$ so that $p_{t}\left(x^{\prime}, y_{0}\right)>0$. Define $a=$ $\frac{1}{2} p_{t}\left(x^{\prime}, y_{0}\right)$ and set $A^{\prime}=\left\{(x, y): p_{t}(x, y) \geq a\right\}$. Since $p_{t}(x, y)$ is continuous in $y$ and $x$, there must be an open ball around $x^{*}$, call it $\mathscr{B}_{1}$, and an open ball around $y_{0}$, call it $A$, so that $\mathcal{B}_{1} \times A \subset A^{\prime}$. Since $A$ is an open ball, $m(A)>0$. Set $\delta=m(A)$ and define $m_{A}(\cdot)$ by

$$
m_{A}(\cdot)=\frac{m(\cdot \cap A)}{\delta} .
$$


Let $\varphi=m_{A}$. We now use the above lemma to show that the diffusion under question has a $\varphi$-irreducible sampled chain.

LEMMA A.4 Let $X_{t}$ be a diffusion satisfying the assumptions of Theorem A.2. Then the sampled chain $\left\{X_{n}^{h}\right\}$ is $\varphi$-irreducible.

Proof: We begin by applying Lemma A.3 with $t=h$ and $x^{\prime}=x^{*}$. We must show that if $m_{A}(B)>0$ then $\mathbb{P}_{x}\left\{X_{n}^{h} \in B\right\}>0$ for some $n$. From Lemma A.3, we know that there exists an open $\mathscr{B}_{1}$ about $x^{*}$ so that if $y \in \mathscr{B}_{2}$ then $\mathbb{P}_{y}\left\{X_{1}^{h} \in B\right\}>$ $\delta m_{A}(B)$. By the second hypothesis of Theorem A.2, for any $x \in \mathbb{R}^{d}$, there exists a $n^{\prime}$ so that $\mathbb{P}_{x}\left\{X_{n^{\prime}}^{h} \in \mathscr{B}_{1}\right\}>0$. Setting $n=n^{\prime}+1$, we have the desired estimate to prove irreducibility. Namely, by the Markov property,

$$
\mathbb{P}_{x}\left\{X_{n}^{h} \in B\right\} \geq \mathbb{P}_{x}\left\{X_{n^{\prime}}^{h} \in \mathscr{B}_{1}\right\} \inf _{y \in \mathscr{B}_{1}} \mathbb{P}_{y}\left\{X_{1}^{h} \in B\right\} \geq \mathbb{P}_{x}\left\{X_{n^{\prime}}^{h} \in \mathcal{B}_{1}\right\} m_{A}(B) .
$$

Since by construction $\mathbb{P}_{x}\left\{X_{n^{\prime}}^{h} \in \mathcal{B}_{1}\right\}>0$, we know that $\mathbb{P}_{x}\left\{X_{n}^{h} \in B\right\}>0$ whenever $m_{A}(B)>0$. Hence, $X_{n}^{h}$ is $\varphi$-irreducible.

Before turning to the proof of Theorem A.2, we recall a last definition and an associated result from [20]. A set $C$ is said to be petite for a Markov chain with transition kernel $P$ if there exists a probability measure $\mu$, a constant $c \in(0,1)$, and a positive sequence $\left\{a_{i}\right\}_{i=1}^{\infty}$ with $\sum a_{i}=1$ such that

$$
\sum_{n=1}^{\infty} a_{n} P^{n}(x, \cdot) \geq c \mu(\cdot) \quad \text { for all } x \in C .
$$

Being petite amounts to a strengthening of the idea of being $\varphi$-irreducible on a set in that it provides a degree of uniformity. This is needed to gain control of the mixing rate. However, as the next lemma shows, in our setting the two ideas are essentially equivalent.

LemMA A.5 Let $X_{t}$ be a nonexploding diffusion in $\mathbb{R}^{d}$ satisfying $(\mathrm{H})$. Consider the Markov chain $X_{n}^{h}$ obtained by sampling with some frequency $h$. Then every compact set is petite for $X_{n}^{h}$ if and only if $X_{n}^{h}$ is $\varphi$-irreducible.

PROOF: This fact is related to the proof of irreducibility given in Lemma A.3. This is simply a combination of theorem 3.3 of [21] and lemma 3.2 from [20]. The result is simply a restatement in different language of the fact that the transition density $p_{t}(x, y)$ is continuous in $x$. The second lemma shows that given this continuity, all compact sets being petite is equivalent to $\varphi$-irreducibility.

We now quote a result from [22] which, together with the above lemmas, will yield Theorem A.2.

THEOREM A.6 (Theorem 5.1 and 6.1 of [22]) Let $X_{t}$ be a right process with a Lyapunov function $V$ as in (L). If all compact sets are petite for some sampled chain, then there exists a positive $B$ and $r$ so that

$$
\left\|P_{t}(x, \cdot)-\pi\right\|_{V} \leq B[1+V(x)] e^{-r t}
$$


with $\mathcal{V}=\{$ measurable $f$ with $|f(x)|<1+V(x)\}$.

Proof of Theorem A.2: Since $X_{t}$ is an Itô diffusion, we know it has a rightcontinuous version. Lemma A.1 establishes the existence of a Lyapunov function. The nonexplosiveness follows easily from the Lyapunov structure (see [11] or theorem 2.1 of [21]), and hence Lemma A.5 implies that all compact sets are petite. Thus Theorem A.6 concludes the proof.

Acknowledgments. The first author's work is partially supported by the National Science Foundation via a Presidential Faculty Fellowship. The second author's work is also partially supported by the National Science Foundation through a postdoctoral fellowship (DMS-9971087). It is a great pleasure to acknowledge the many helpful comments by our close collaborator, Yasha Sinai. We would also like to acknowledge stimulating conversations with Gérard Ben Arous, Amir Dembo, George Papanicolaou, Andrew Stuart, and S. R. S. Varadhan.

\section{Bibliography}

[1] Azéma, J.; Duflo, M.; Revuz, D. Propriétés relatives des processus de Markov récurrents. Z. Wahrscheinlichkeitstheorie und Verw. Gebiete. 13 (1969), 286-314.

[2] Bricmont, J.; Kupiainen, A.; Lefevere, R. Preprint, 2000.

[3] Constantin, P.; Foias, C. Navier-Stokes equations. Chicago Lectures in Mathematics. University of Chicago Press, Chicago, 1988.

[4] E, W.; Mattingly, J. C.; Sinai, Ya. G. Gibbsian dynamics and ergodicity for the stochastic forced Navier-Stokes equation. Preprint, 2000.

[5] E, W.; Vanden Eijnden, E. Remarks on two-dimensional turbulence. Preprint.

[6] Eckmann, J. P.; Hairer, M. Uniqueness of the invariant measure for a stochastic pde driven by degenerate noise. Preprint.

[7] Ferrario, B. Ergodic results for stochastic Navier-Stokes equation. Stochastics Stochastics Rep. 60 (1997), no. 3-4, 271-288.

[8] Flandoli, F.; Maslowski, B. Ergodicity of the 2-D Navier-Stokes equation under random perturbations. Comm. Math. Phys. 172 (1995), no. 1, 119-141.

[9] Getoor, R. K. Transience and recurrence of Markov processes. Seminar on Probability, XIV (Paris, 1978/1979), 397-409. Lecture Notes in Mathematics, 784. Springer, Berlin, 1980.

[10] Harris, T. E. The existence of stationary measures for certain Markov processes. Proceedings of the Third Berkeley Symposium on Mathematical Statistics and Probability, 1954-1955, vol. II, 113-124. University of California Press, Berkeley and Los Angeles, 1956.

[11] Has'minskiǔ, R. Z. Stochastic stability of differential equations. Monographs and Textbooks on Mechanics of Solids and Fluids: Mechanics and Analysis, 7. Sijthoff and Noordhoff, Alphen aan den Rijn, Germantown, Md., 1980.

[12] Ichihara, K.; Kunita, H. A classification of the second order degenerate elliptic operators and its probabilistic characterization. Z. Wahrscheinlichkeitstheorie und Verw. Gebiete 30 (1974), 235-254.

[13] Kliemann, W. Recurrence and invariant measures for degenerate diffusions. Ann. Probab. 15 (1987), no. 2, 690-707.

[14] Kuksin, S.; Shirikyan, A. Stochastic dissipative PDEs and Gibbs measures. Comm. Math. Phys. 213 (2000), no. 2, 291-330. 
[15] Kunita, H. Supports of diffusion processes and controllability problems. Proceedings of the International Symposium on Stochastic Differential Equations (Res. Inst. Math. Sci., Kyoto Univ., Kyoto, 1976), 163-185. Wiley, New York-Chichester-Brisbane, 1978.

[16] Kunita, H. Stochastic flows and stochastic differential equations. Cambridge Studies in Advanced Mathematics, 24. Cambridge University Press, Cambridge, 1990.

[17] Kusuoka, S.; Stroock, D. Applications of the Malliavin calculus. I. II. Stochastic analysis (Katata/Kyoto, 1982), 271-306. North-Holland Mathematical Library, 32. North-Holland, Amsterdam, 1984; J. Fac. Sci. Univ. Tokyo Sect. IA Math. 32 (1985), no. 1, 1-76.

[18] Mattingly, J. C. Ergodicity of 2D Navier-Stokes equations with random forcing and large viscosity. Comm. Math. Phys. 206 (1999), no. 2, 273-288.

[19] Mattingly, J. C.; Stuart, A. Ergodicity for sdes: Locally lipschitz vector fields and degenerate noise. Submitted, 2000.

[20] Meyn, S. P.; Tweedie, R. L. Stability of Markovian processes. I. Criteria for discrete-time chains. Adv. in Appl. Probab. 24 (1992), no. 3, 542-574.

[21] Meyn, S. P.; Tweedie, R. L. Stability of Markovian processes. II. Continuous-time processes and sampled chains. Adv. in Appl. Probab. 25 (1993), no. 3, 487-517.

[22] Meyn, S. P.; Tweedie, R. L. Stability of Markovian processes. III. Foster-Lyapunov criteria for continuous-time processes. Adv. in Appl. Probab. 25 (1993), no. 3, 518-548.

[23] Norris, J. Simplified Malliavin calculus. Séminaire de Probabilités, XX, 1984/85, 101-130. Lecture Notes in Mathematics, 1204. Springer, Berlin, 1986.

WEINAN E

Princeton University

Department of Mathematics

Program in Applied and Computational Mathematics

Fine Hall, Washington Road

Princeton, NJ 08544-1000

E-mail: weinane

math.princeton.edu

and

Peking University

School of Mathematical Sciences

Beijing 100871

PEOPLE'S REPUBLIC OF CHINA

Received December 2000.
JONATHAN C. MATTINGLY

Stanford University

Department of Mathematics

Building 380, MC 2125

Stanford, CA 94305-2125

E-mail: jonmemath.stanford.edu 